\title{
Research on the Construction of Large Data Management Platform of Medical Association
}

\author{
Jinguo Wang ${ }^{1, ~ a}$ and corresponding author: Na Wang ${ }^{2, b}$ \\ ${ }^{1}$ Department of Urology, The First Hospital of Jilin University, Changchun, China \\ ${ }^{2}$ Department of Anesthesiology, The First Hospital of Jilin University, Changchun, China \\ awangjinguolily@163.com, bwangna080613@163.com
}

\begin{abstract}
Keywords: Construction, Large data, Management, Platform, Medical association.
Abstract. In highly-developed big data age, how to cover different departments and different management systems of various ownership and how to integrate a variety of medical modes are the most important problems to solve in the current medical model needs. It can achieve the optimal combination of the medical resources and effective use. It becomes standard data aggregation and distribution platform, constructing medical big data platform as medical support platform and setting up a standard system, unified data collection, indicators caliber and data interface standard. The platform provides a customized application model library for the medical association, and it is easy to realize the independent design that conforms to the hospital's own medical mode. By using big data analysis and mining technology to build intelligent hierarchical diagnosis and treatment system and expert database, better balance, medical resources can be shared and optimized.
\end{abstract}

\section{Introduction}

In recent years, Chinese health data have grown exponentially, giving rise to a number of start-ups and more scientific support. But its value has not been effectively mined, and there is even a tendency to fall into a sea of data without direction. A number of medical institutions did not keep up with the pace of the development. Through channels of big data associated with medical health, for medical science there is a huge value, but most of the construction and the regulators. [1] Use of big data analytics pathology and etiology of disease will also find other opportunities, such as lung cancer and gastrointestinal cancer incidence is higher in China, looking for these samples and analysis research and development goal of pharmaceutical enterprises is extremely valuable.

Since the reform of public hospitals has no measurement and value scale, the goal is clear, but it is not a solid result. Public hospital reform to today, the most absent is the basic medical data and facilities. Database construction began to be out of place, mainly in the resources, now the most vacant is management idea, management ideas and management methods. [2]

\section{Electronic medical records of clinical research integration}

The standardization of electronic medical records is the foundation of the integration of clinical research, which is an important guarantee for the realization of data sharing and utilization. To reform the clinical data for standardization can improve the existing system in the lack of necessary data, improve the level of the existing data collection of structured, realize outpatient, hospital, follow-up, patients with physical examination and other comprehensive medical treatment and prognostic information integration. [3] Follow the standardized method of transformation of the standardization of electronic medical records, transformation process includes: analysis of the existing medical record templates data elements to determine the template name, code, define the data the corresponding domain name, code, data - data warehousing - create a new template.

The standardization of electronic medical records reform process need to clinical doctors spend a lot of time and energy, and information technology personnel communication and discussion, over and over again from the aspects of electronic medical records meet the demand of scientific research and practical usability comprehensive consideration, determine the need to transform the data and template 
content presentation, to guarantee the electronic medical record templates after transforming real integrates clinical scientific research. [4]

Template transformation, for example, the template content is divided into 10 data segments, including chief complaint, examination of the history of menstruation, past medical history, personal history, physical examination, specialized examination, rating, auxiliary examination, processing, has established standardized data, in addition to the chief complaint, auxiliary examination and treatment in the rest of the content are optional, convenient for data acquisition and laid a solid foundation for scientific research and using data. [5]

\section{Data model and application mode}

Data Processing Model. The medical data in a big data platform form the hospital information platform, according to the relevant business application after consolidation. It can be used for processing data for medical staff, patients and hospital management.

Data Acquisition. The data acquisition is to obtain relevant raw data from the hospital information platform from the hospital information platform according to the application subject and store it in the database of medical big data platform. [6]

Mainly rely on the existing empirical judgment to run a major administrative decision-making social stability risk assessment system, in today's data explosion, a diversified era of big data, just rely on the traditional way to collect the number of scarce, limited coverage, aging is insufficient data to meet the scientific standard of social stability risk assessment, this also is many administrative decision is social stability risk assessment but it still can't to ensure scientific decision-making of the important reasons. [7]

Data integration. The data integration is the integration of business data extracted from the hospital information platform according to the unified storage and definition. After years of development, hospital informatization has accumulated many basic and scattered business data. But the data scattered in different departments such as clinical, medical and management, the data query access difficulties, the hospital management personnel can't directly access to data if the data were analyzed using, need data of different formats and business systems. [8]

Data Processing. Data processing will be used to clean, transform and load the data, and establish the model to calculate and aggregate the data according to the business rules. Therefore, to review the connotation of the social stability risk assessment system of major administrative decision-making in the perspective of big data will make its own assessment dilemma very clear.

Data Presentation. The data presentation is the data visualization, which is to facilitate the understanding and reading of data by medical staff, patients and managers, and data conversion based on relevant technologies. [9]

The interconnection between mobile Internet medical application system and traditional medical service system needs to be solved. Whether to medical personnel as the main body in the operation, such as mobile rounds workstation, nurse workstation, movement of outpatient infusion system, etc., as a main body or in the patients with operation, such as make an appointment, report query, line up your turn, mobile payment, health advice, such as a mobile application, must be with hospital HIS existing, inspection inspection information system for data exchange or business together. [10]

\section{Data Analysis Model}

Data analysis model medical data can serve patients, clinical medical care and hospital management. Health big data analysis platform and application through field investigation and deep interview research project, combined with the existing medical big data research, demand for medical applications of big data fully analyzed and summarized, based on process to the patient's medical data analysis and application of the model. [11] The model shows from a patient's admission to discharge 
process of the relevant data, including patient characteristics, disease data, treatment plan and cost data, data and treatment status in the process of data management.

Inspection examination data, for example, the doctor issued by inspection and examination application information, and test results, reports, and check the information in the form of structured documents stored in the platform, patients can query at any time by PC and mobile medical application platform on the related test results. The demand for personal health services provided by mobile medical services is matched by the ongoing hospital management and interactive business. Through mobile APP application, we can interact with medical information, such as separation, payment result, drug withdrawal, result query, etc.

Outside the court to provide patient portal, so it can continue to enjoy medical treatment service, including pertinent health education on knowledge, their own school health monitoring information, medication appointment reminders, remote medical consultation, visits, etc., make broad residents can detect ecg, blood sugar testing at home, at the same time get the professional feedback in time. [12] With the aid of information platform, integrated health life cycle all diagnostic information, including all kinds of test results, radiation check images, prescription, follow-up, and health monitoring data information, such as clinical medical service personnel can timely understanding of service object complete medical activity, is helpful to improve the level of medical treatment, to reduce the medical risk.

\section{Conclusions}

Ideas determine ideas. In combing the health outstanding problems at the same time, deep understanding of health medical applications of big data will give economic and social development. Health care mode and people's life style and so on various aspects brought extensive and profound changes. To conform to the development and change, change ideas and idea renewal must be pilot.

The introduction of big data analysis technology can deeply dig deep information of data, reveal the problems existing in the hidden system of data behind the data, and the core factors that influence the advancement of the system. By analyzing the correlation between data, the whole data flow and its externality is fully utilized.

\section{References}

[1] Department of The National Program for IT in the NHS. Twentieth Report of Session 2006-2007. 2007

[2] Gibson M.J.S, Gregory S.R, Pandya S.M. et al. Long-term Care in Developed Nations. A Brief Overview. 2003

[3] Ex vivo expanded mobilized peripheral blood CD34 cells accelerate haematological recovery in a baboon model of autologous transplantation[J]. British Journal of Haematology. 2008 (1)

[4] Fabio Efficace, Markus Horneber, Stephane Lejeune, Frits Van Dam, Suzanne Leering, Michael Rottmann, Neil K. Aaronson. Methodological quality of patient-reported outcome research was low in complementary and alternative medicine in oncology[J]. Journal of Clinical Epidemiology. 2006 (12)

[4] IngelaWiklund. Assessment of patient $\square$ reported outcomes in clinical trials: the example of health-related quality of life[J]. Fundamental \& Clinical Pharmacology. 2004 (3)

[5] Group for the respect of ethics, excellence in science (GREES): rheumatoid arthritis sectionlDM

Reid. Report. Recommendations for the registration of drugs used in the treatment of rheumatoid arthritis[J]. British Journal of Rheumatology. 1998

[6] McCall Kathryn, Keen Jeremy, Farrer Keith, Maguire Roma, McCann Lisa, Johnston Bridget, McGill Maria, Sage Meurig, Kearney Nora. Perceptions of the use of a remote monitoring system in patients receiving palliative care at home. International journal of palliative nursing. 2008

[7] Jaana Mirou, Paré Guy, Sicotte Claude. Home telemonitoring for respiratory conditions: a systematic review. The American journal of managed care. 2009 
[8] Shahriyar R., Bari F., Akbar M., Kundu G., Ahamed S.I. Intelligent mobile health monitoring system (IMHMS). International Journal of Control and Automation. 2009

[9] Jeffrey S. Levin, Thomas A. Glass, Lawrence H. Kushi,John R. Schuck, Lea Steele, Wayne B. Jonas. Quantitative Methods in Research on Complementary and Alternative Medicine: A Methodological Manifesto[J]. Medical Care. 1997 (11)

[10] Anders Carlberg. Patient satisfaction and design of treatment: Results from a study of two different ways of treating hypertension[J]. Scandinavian Journal of Primary Health Care. 1993 (2) [11] Nanette K. Wenger. Assessment of quality of life: A medical imperative[J]. Cardiovascular Drugs and Therapy. 1988 (5)

[12] Gund A, Ekman I, Lindecrantz K, Sjoqvist B A, Staaf E L, Thorneskold N. Design evaluation of a home-based telecare system for Chronic Heart Failure patients. Conference proceedings: Annual International Conference of the IEEE Engineering in Medicine and Biology Society. IEEE Engineering in Medicine and Biology Society. Conference. 2009 